\title{
On logarithmic residue of monogenic functions in a three-dimensional commutative algebra with one-dimensional radical
}

\author{
R. Pukhtaievych and S. Plaksa
}

\begin{abstract}
We consider monogenic functions taking values in a three-dimensional commutative algebra $\mathbb{A}_{2}$ over the field of complex numbers with onedimensional radical. We calculate the logarithmic residues of monogenic functions acting from a three-dimensional real subspace of $\mathbb{A}_{2}$ into $\mathbb{A}_{2}$. It is shown that the logarithmic residue depends not only on zeros and singular points of a function but also on points at which the function takes values in ideals of $\mathbb{A}_{2}$, and, in general case, is a hypercomplex number.
\end{abstract}

\section{Introduction.}

The logarithmic residue in a Banach algebra means a contour integral of the logarithmic derivative of a hypercomplex function. It was considered by many authors in many algebras, for instance, an algebra of all bounded linear operators on a complex Banach space (see, e.g., $[1,2,3]$ ), matrix algebras (see, e.g., [4]), a biharmonic algebra (see, e.g., [5]), a three-dimensional algebra with twodimensional radical (see, e.g., [6]). We first mention Bart [7]. He considered the logarithmic residue for functions acting from the field of complex numbers $\mathbb{C}$ to a commutative Banach algebra. One of the main issue considered in [7] (see also [2], [3]) is whether vanishing of a logarithmic residue implies that a function takes only invertible values inside an integration contour, where

Key Words: Commutative Banach algebra, monogenic function, logarithmic residue 2010 Mathematics Subject Classification: Primary 30G35; Secondary 32A27.

Received: 20.12.2016

Accepted: 20.02.2017 
contours are considered on the complex plain. We note that the answer is negative in general case.

We also mention Bart, Ehrhardt and Silbermann [8] which considered the logarithmic residues of locally analytic and meromorphic functions $f$ given in bounded Cauchy domains in the complex plane and taking values in a Banach algebra $\mathbb{A}$ with a unit element over $\mathbb{C}$. For definitions of mentioned functions and domains, we refer, e.g., to Taylor [9, sec. V.1.]. For instance, if $f: \mathbb{C} \rightarrow \mathbb{A}$ is an analytic or meromorphic function in a bounded Cauchy domain in $\mathbb{C}$ then the logarithmic residue of $f$ is equal to a linear combination of idempotents of $\mathbb{A}$ with integer coefficients (see [8, Thm. 6.1, Thm. 7.1]). For the residues in multidimensional complex analysis and their applications we refer to $[10,11,12]$.

The logarithmic residues of monogenic functions (i.e., continuous and Gateaux differentiable) were considered in [5] and [6]. For instance, there was calculated the logarithmic residue of monogenic function and it was shown that it is always an integer number. In general case, it can be a hypercomplex number.

This paper is concerned with the logarithmic residues of monogenic functions taking values in a three-dimensional commutative associative Banach algebra $\mathbb{A}_{2}$ over $\mathbb{C}$ with one-dimensional radical. We calculate the logarithmic residues of monogenic functions which act from a three-dimensional real subspace of $\mathbb{A}_{2}$ into $\mathbb{A}_{2}$.

Now, we briefly outline an organizing of the article. In section 2, we introduce some standard notations. In section 3 , we consider some properties of Laurent series of monogenic functions in $\mathbb{A}_{2}$. Section 4 is devoted to the logarithmic residues. In this section, we exploit the Laurent series to calculate the logarithmic residue of monogenic function (see Lemma 4.5). Using this result, we establish the validity of Theorem 4.7 and Theorem 4.8 for a curvilinear integral of the logarithmic derivative of a monogenic function along a family of curves. At the end of this article, we have enclosed Appendix A with some results exploited in the paper.

We also note, the strategy applied in section 4 can be applied to compute the logarithmic residue of monogenic functions taking values in an arbitrary finite-dimensional commutative algebra.

\section{Preliminaries and notations}

Throughout this paper $\mathbb{R}$ and $\mathbb{C}$ denote the fields of real and complex numbers, respectively.

Let $\mathbb{A}_{2}$ be a three-dimensional commutative associative Banach algebra over $\mathbb{C}$ with one-dimensional radical. This algebra has a basis $\left\{I_{1}, I_{2}, \rho\right\}$ with 
the following multiplication rules for its elements

$$
I_{1}^{2}=I_{1}, \quad I_{2}^{2}=I_{2}, \quad I_{2} \rho=\rho, \quad I_{1} I_{2}=\rho^{2}=I_{1} \rho=0 .
$$

The unit of $\mathbb{A}_{2}$ is represented as $1=I_{1}+I_{2}$.

There are two maximal ideals in $\mathbb{A}_{2}$ :

$$
\mathcal{J}_{1}:=\left\{t_{1} I_{2}+t_{2} \rho: t_{1}, t_{2} \in \mathbb{C}\right\}, \quad \mathcal{J}_{2}:=\left\{t_{1} I_{1}+t_{2} \rho: t_{1}, t_{2} \in \mathbb{C}\right\} .
$$

Both ideals together include all noninvertible elements of the algebra $\mathbb{A}_{2}$ and consist of such elements only. The radical of algebra is the set $\mathcal{R}=\mathcal{J}_{1} \cap \mathfrak{J}_{2}$.

Let $c=c_{1} I_{1}+c_{2} I_{2}+c_{3} \rho$, where $c_{1}, c_{2}, c_{3} \in \mathbb{C}$. The element $c$ is invertible if and only if $c_{1} \neq 0$ and $c_{2} \neq 0$, moreover, the inverse element $c^{-1}$ is represented as

$$
c^{-1}=\frac{1}{c_{1}} I_{1}+\frac{1}{c_{2}} I_{2}-\frac{c_{3}}{c_{2}^{2}} \rho .
$$

The logarithm is defined in [13, p. 422] and takes the following form in the basis $\left\{I_{1}, I_{2}, \rho\right\}$

$$
\ln c:=\left(\ln c_{1}\right) I_{1}+\left(\ln c_{2}\right) I_{2}+\frac{c_{3}}{c_{2}} \rho,
$$

where $\ln c_{1}, \ln c_{2}$ are principal branches of appropriate logarithmic functions.

We consider the linear continuous functionals $f_{1}, f_{2}: \mathbb{A}_{2} \rightarrow \mathbb{C}$ satisfying the equalities

$$
f_{1}\left(I_{1}\right)=f_{2}\left(I_{2}\right)=1, \quad f_{1}\left(I_{2}\right)=f_{1}(\rho)=f_{2}\left(I_{1}\right)=f_{2}(\rho)=0 .
$$

The maximal ideals $\mathfrak{J}_{1}, J_{2}$ are kernels of functionals $f_{1}, f_{2}$, respectively. For an arbitrary set $X$ in $\mathbb{A}_{2}$, we find it convenient to set

$$
\begin{aligned}
& D_{1}(X):=\left\{\xi \in \mathbb{C}: \xi=f_{1}(a) \forall a \in X\right\}, \\
& D_{2}(X):=\left\{\xi \in \mathbb{C}: \xi=f_{2}(a) \forall a \in X\right\} .
\end{aligned}
$$

Let $e_{1}, e_{2}, e_{3}$ be vectors which are linear independent over $\mathbb{R}$ and have the following form

$$
e_{1}=1, \quad e_{2}=p_{1} I_{1}+p_{2} I_{2}+p_{3} \rho, \quad e_{3}=q_{1} I_{1}+q_{2} I_{2}+q_{3} \rho,
$$

where $p_{k}, q_{k} \in \mathbb{C}$ for all $k \in\{1,2,3\}$.

The norm in $\mathbb{A}_{2}$ is defined for all $a:=a_{1} e_{1}+a_{2} e_{2}+a_{3} e_{3} \in \mathbb{A}_{2}$, where $a_{1}, a_{2}, a_{3} \in \mathbb{C}$, in the following way

$$
\|a\|:=\sqrt{\left|a_{1}\right|^{2}+\left|a_{2}\right|^{2}+\left|a_{3}\right|^{2}} .
$$


Let $E_{3}$ be a linear span over $\mathbb{R}$, which is generated by the vectors of basis $\left\{e_{1}=1, e_{2}, e_{3}\right\}$ and defined by

$$
E_{3}:=\left\{\zeta=x e_{1}+y e_{2}+z e_{3}: x, y, z \in \mathbb{R}\right\}
$$

such that $D_{k}\left(E_{3}\right)=\mathbb{C}$ for all $k \in\{1,2\}$. Obviously, it holds if and only if at least one of the numbers $p_{k}$ or $q_{k}$ belongs to $\mathbb{C} \backslash \mathbb{R}$ for all $k \in\{1,2\}$.

In what follows, $x, y, z \in \mathbb{R}$ and $x_{0}, y_{0}, z_{0} \in \mathbb{R}$. Let $\zeta:=x e_{1}+y e_{2}+z e_{3}$ and $\zeta_{0}:=x_{0} e_{1}+y_{0} e_{2}+z_{0} e_{3}$. We set

$$
\begin{array}{lr}
\xi_{1}:=x+p_{1} y+q_{1} z, & \xi_{10}:=x_{0}+p_{1} y_{0}+q_{1} z_{0}, \\
\xi_{2}:=x+p_{2} y+q_{2} z, & \xi_{20}:=x_{0}+p_{2} y_{0}+q_{2} z_{0}, \\
e_{1}^{*}:=\left(\operatorname{Re} p_{1} \operatorname{Im} q_{1}-\operatorname{Im} p_{1} \operatorname{Re} q_{1}\right) e_{1}-\operatorname{Im} q_{1} e_{2}+\operatorname{Im} p_{1} e_{3}, \\
e_{2}^{*}:=\left(\operatorname{Re} p_{2} \operatorname{Im} q_{2}-\operatorname{Im} p_{2} \operatorname{Re} q_{2}\right) e_{1}-\operatorname{Im} q_{2} e_{2}+\operatorname{Im} p_{2} e_{3}, \\
L_{1}(\zeta):=\left\{\zeta+t e_{1}^{*}: t \in \mathbb{R}\right\}, & L_{2}(\zeta):=\left\{\zeta+t e_{2}^{*}: t \in \mathbb{R}\right\} .
\end{array}
$$

We say that a domain $\Omega$ is convex in the direction $L_{1}$ (or $L_{2}$ ) if a set $\Omega \cap L_{1}(\zeta)$ (or $\Omega \cap L_{2}(\zeta)$ ) is connected for all $\zeta \in \Omega$.

For two arbitrary vectors $a, b \in E_{3}$ defined as $a=a_{1} e_{1}+a_{2} e_{2}+a_{3} e_{3}$, $b=b_{1} e_{1}+b_{2} e_{2}+b_{3} e_{3}$, we denote

$$
a \times b:=\left(a_{2} b_{3}-a_{3} b_{2}\right) e_{1}+\left(a_{3} b_{1}-a_{1} b_{3}\right) e_{2}+\left(a_{1} b_{2}-a_{2} b_{1}\right) e_{3} .
$$

We find it convenient to set

$$
\begin{aligned}
& \hat{e}_{1}:=\left\{\begin{array}{lll}
e_{1}^{*} \times e_{1} & \text { if } & e_{1}^{*}=e_{2}^{*}, \\
e_{1}^{*} \times e_{2}^{*} & \text { if } & e_{1}^{*} \neq e_{2}^{*},
\end{array}\right.
\end{aligned}
$$

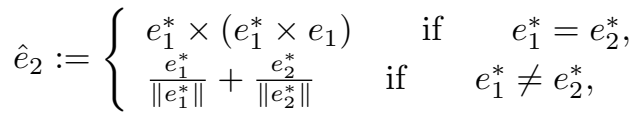

$$
\begin{aligned}
& \Pi(\zeta):=\left\{\zeta+t_{1} \hat{e}_{1}+t_{2} \hat{e}_{2}: t_{1}, t_{2} \in \mathbb{R}\right\} .
\end{aligned}
$$

Let $\Omega$ be a domain in $E_{3}$. We say that a continuous function $\Phi: \Omega \rightarrow \mathbb{A}_{2}$ is monogenic in $\Omega$ if $\Phi$ is differentiable in the sense of Gateaux in every point of $\Omega$, i.e., if for every $\zeta \in \Omega$ there exists an element $\Phi^{\prime}(\zeta) \in \mathbb{A}_{2}$ such that

$$
\lim _{\varepsilon \rightarrow 0+0}(\Phi(\zeta+\varepsilon h)-\Phi(\zeta)) \varepsilon^{-1}=h \Phi^{\prime}(\zeta) \quad \forall h \in E_{3} .
$$

$\Phi^{\prime}(\zeta)$ is the Gateaux derivative of a function $\Phi$ at the point $\zeta$. 
We also set

$$
\begin{array}{r}
\mathcal{K}_{r, R}\left(\zeta_{0}\right):=\left\{\zeta \in E_{3}: 0 \leq r<\left|\xi_{1}-\xi_{10}\right|<R \leq \infty,\right. \\
\left.0 \leq r<\left|\xi_{2}-\xi_{20}\right|<R \leq \infty\right\}, \\
\mathcal{K}_{R}\left(\zeta_{0}\right):=\left\{\zeta \in E_{3}:\left|\xi_{1}-\xi_{10}\right|<R,\left|\xi_{2}-\xi_{20}\right|<R\right\} .
\end{array}
$$

If a domain $\Omega \subset E_{3}$ is convex in both directions $L_{1}$ and $L_{2}$, then every monogenic function $\Phi: \Omega \rightarrow \mathbb{A}_{2}$ can be expressed in the form

$$
\Phi(\zeta)=F_{1}\left(\xi_{1}\right) I_{1}+F_{2}\left(\xi_{2}\right) I_{2}+\left(\left(p_{3} y+q_{3} z\right) F_{2}^{\prime}\left(\xi_{2}\right)+F_{0}\left(\xi_{2}\right)\right) \rho \quad \forall \zeta \in \Omega,
$$

where $F_{1}$ is a holomorphic function in the domain $D_{1}(\Omega)$ and $F_{2}, F_{0}$ are holomorphic functions in the domain $D_{2}(\Omega)$ (cf. [15, Thm. 5.4]). We note that representation (2) is also proved in [14, Thm. 4] for the case where the basis $\left\{e_{1}, e_{2}, e_{3}\right\}$ is harmonic.

\section{Properties of Laurent series of monogenic functions in $\mathbb{A}_{2}$}

Let $\zeta_{0}$ be a point in a domain $\Omega \subset E_{3}$. In a neighbourhood of $\zeta_{0}$ contained in $\Omega$ let us take a circle $C\left(\zeta_{0}\right)$ with the center at the point $\zeta_{0}$. Let $k \in\{1,2\}$. We say that the circle $C\left(\zeta_{0}\right)$ surrounds the set $L_{k}\left(\zeta_{0}\right)$ if $D_{k}\left(C\left(\zeta_{0}\right)\right)$ bounds a domain $D_{k}^{\prime}$ and $f_{k}\left(\zeta_{0}\right) \in D_{k}^{\prime}$. We say that the curve $\gamma \subset \Omega$ surrounds once the set $L_{k}\left(\zeta_{0}\right)$ if there exists a circle $C\left(\zeta_{0}\right) \subset \Omega$ which surrounds the mentioned set and is homotopic to $\gamma$ in the domain $\Omega \backslash L_{k}\left(\zeta_{0}\right)$.

Every monogenic in $\mathcal{K}_{0, R}\left(\zeta_{0}\right)$ function $\Phi$ can be represented as the sum of convergent Laurent series (see [16, Thm. 3])

$$
\Phi(\zeta)=\sum_{n=-\infty}^{\infty} d_{n}\left(\zeta-\zeta_{0}\right)^{n}
$$

where $\left(\zeta-\zeta_{0}\right)^{n}:=\left(\left(\zeta-\zeta_{0}\right)^{-1}\right)^{-n}$ for $n=-1,-2, \ldots$ and the coefficients $d_{n}$ are defined as

$$
d_{n}=a_{n} I_{1}+b_{n} I_{2}+\left((n+1)\left(p_{3} y_{0}+q_{3} z_{0}\right) b_{n+1}+c_{n}\right) \rho,
$$

where $a_{n}, b_{n}, c_{n}$ are coefficients of Laurent series of the functions in decomposition (2):

$$
\begin{gathered}
F_{1}\left(\xi_{1}\right)=\sum_{n=-\infty}^{\infty} a_{n}\left(\xi_{1}-\xi_{10}\right)^{n}, \quad F_{2}\left(\xi_{2}\right)=\sum_{n=-\infty}^{\infty} b_{n}\left(\xi_{2}-\xi_{20}\right)^{n}, \\
F_{0}\left(\xi_{2}\right)=\sum_{n=-\infty}^{\infty} c_{n}\left(\xi_{2}-\xi_{20}\right)^{n}
\end{gathered}
$$


Furthermore, the coefficients $d_{n}$ can be represented (as in [17, Thm. 4]) by the formula

$$
d_{n}=\frac{1}{2 \pi i} \int_{\gamma} \Phi(\tau)\left(\left(\zeta-\zeta_{0}\right)^{-1}\right)^{n+1} d \tau, \quad n=0, \pm 1, \pm 2, \ldots
$$

where $\gamma$ is an arbitrary closed Jordan rectifiable curve in $\mathcal{K}_{0, R}\left(\zeta_{0}\right)$ surrounding once the straight lines $L_{1}\left(\zeta_{0}\right), L_{2}\left(\zeta_{0}\right)$.

Remark. It should be noted that this result is proved in [16, Thm. 3] but the proof is actually done under the assumption of convexity of the domain $\mathcal{K}_{0, R}\left(\zeta_{0}\right)$ in directions $L_{1}$ and $L_{2}$. However, the statement of Theorem 3 in [16] is true. It follows from the following considerations. By Lemma A.1 in Appendix $\mathrm{A}$, the domain $\mathcal{K}_{0, R}\left(\zeta_{0}\right)$ can be represented as a union of domains $\widetilde{\mathcal{K}}_{0, R}\left(\zeta_{0}\right), \widehat{\mathcal{K}}_{0, R}\left(\zeta_{0}\right)$ which are convex in both directions $L_{1}$ and $L_{2}$, and $\widetilde{\mathcal{K}}_{0, R}\left(\zeta_{0}\right) \cap \widehat{\mathcal{K}}_{0, R}\left(\zeta_{0}\right)$ is an open set. Then, the monogenic function $\Phi$ has representation (3) in both domains $\widetilde{\mathcal{K}}_{0, R}\left(\zeta_{0}\right)$ and $\widehat{\mathcal{K}}_{0, R}\left(\zeta_{0}\right)$, and, as a conclusion of [16, Thm. 2], has the same representation in $\mathcal{K}_{0, R}\left(\zeta_{0}\right)$.

Next, we define types of singular points of a monogenic function $\Phi$. Namely, the point $\zeta_{0}$ is called:

- a removable singularity of $\Phi$ if the exists a finite limit

$$
\lim _{\zeta \rightarrow \zeta_{0}, \zeta \notin L_{1}\left(\zeta_{0}\right) \cup L_{2}\left(\zeta_{0}\right)} \Phi(\zeta)=A ;
$$

- a pole of $\Phi$ if the exists an infinite limit

$$
\lim _{\zeta \rightarrow \zeta_{0}, \zeta \notin L_{1}\left(\zeta_{0}\right) \cup L_{2}\left(\zeta_{0}\right)} \Phi(\zeta)=\infty ;
$$

- an essential singularity of $\Phi$ if a limit of $\Phi(\zeta)$ does not exist as $\zeta \rightarrow \zeta_{0}$ and $\zeta \notin L_{1}\left(\zeta_{0}\right) \cup L_{2}\left(\zeta_{0}\right)$.

It is known that the isolated singularity can be only removable. Otherwise, if $\Phi$ has a non-removable singularity at the point $\zeta_{0} \in \Omega$, all points of the set $\Omega \cap L_{1}\left(\zeta_{0}\right)$ or the set $\Omega \cap L_{2}\left(\zeta_{0}\right)$, or both these sets are singular for $\Phi$ (cf., [16, sec. 3])

\section{Logarithmic residue of monogenic functions in the al- gebra $\mathbb{A}_{2}$}

Let $\Phi: \mathcal{K}_{0, R}\left(\zeta_{0}\right) \rightarrow \mathbb{A}_{2}$ be a monogenic function in the domain $\mathcal{K}_{0, R}\left(\zeta_{0}\right)$. If $\Phi^{\prime}(\zeta)(\Phi(\zeta))^{-1}$ is monogenic in $\mathcal{K}_{0, R}\left(\zeta_{0}\right)$, the logarithmic residue of the func- 
tion $\Phi$ at the point $\zeta_{0}$ is defined as

$$
\frac{1}{2 \pi i} \int_{\Gamma_{r}\left(\zeta_{0}\right)} \Phi^{\prime}(\zeta)(\Phi(\zeta))^{-1} d \zeta
$$

where $0<r<R$ and

$$
\Gamma_{r}\left(\zeta_{0}\right):=\left\{\zeta \in \Pi\left(\zeta_{0}\right):\left|\xi_{10}-f_{1}(\zeta)\right|=r,\left|\xi_{20}-f_{2}(\zeta)\right|=r\right\}
$$

By virtue of Theorem A.3 in Appendix A, we conclude that the value of the logarithmic residue is independent of $r$ for $0<r<R$.

Clearly, it is reasonable to consider the logarithmic residue not only at zeros and singular points of the function $\Phi$ but also at points where the values of function $\Phi$ belong to the ideals of $\mathbb{A}_{2}$.

We state a necessary and sufficient condition for the existence of integral (5) in the following lemma.

Lemma 4.1. Let $\Phi: \mathcal{K}_{0, R}\left(\zeta_{0}\right) \rightarrow \mathbb{A}_{2}$ be a monogenic function in the domain $\mathcal{K}_{0, R}\left(\zeta_{0}\right)$. The following statements are equivalent:

(i) There exists $R_{1}<R$ such that integral (5) exists for all $0<r<R_{1}$.

(ii) There exists $R_{2}<R$ such that $\Phi(\zeta) \notin \mathcal{J}_{1} \cup \mathcal{J}_{2}$ for all $\zeta \in \mathcal{K}_{0, R_{2}}\left(\zeta_{0}\right)$.

Proof. First, we prove that $(i)$ implies $(i i)$. Assume, for the sake of contradiction, that the statement does not hold. In other words, for all arbitrarily small $R_{2}>0$ there exist points $\zeta \in \mathcal{K}_{0, R_{2}}\left(\zeta_{0}\right)$ for which $\Phi(\zeta) \in \mathcal{J}_{k}, k \in\{1,2\}$. Then the inner point $\xi_{k 0}$ of the domain $D_{k}\left(\mathcal{K}_{R_{2}}\left(\zeta_{0}\right)\right)$ is a limit point of the set of zeros of the holomorphic function $F_{k}$ appearing in equality (2). Hence, according to the uniqueness theorem for holomorphic functions of a complex variable [18, p. 209], $F_{k} \equiv 0$ and, in view of equality (2), we conclude that all values of the function $\Phi$ belong to the ideal $\mathcal{J}_{k}$. Therefore, integral (5) does not exist and we have a contradiction.

Now, we prove that $(i i)$ implies $(i)$. It is enough to note that the assumption $\Phi(\zeta) \notin \mathcal{J}_{1} \cup \mathcal{J}_{2}$ for all $\zeta \in \mathcal{K}_{0, R_{2}}\left(\zeta_{0}\right)$ implies that $\Phi^{\prime}(\zeta)(\Phi(\zeta))^{-1}$ exists for all $\zeta \in \mathcal{K}_{0, R_{2}}\left(\zeta_{0}\right)$ and is monogenic in $\mathcal{K}_{0, R_{2}}\left(\zeta_{0}\right)$. We set $R_{1}=R_{2}$ and, thus, integral (5) exists for all $0<r<R_{1}$.

The following result holds.

Lemma 4.2. Let $\Phi: \mathcal{K}_{0, R}\left(\zeta_{0}\right) \rightarrow \mathbb{A}_{2}$ be a monogenic function in the domain $\mathcal{K}_{0, R}\left(\zeta_{0}\right)$ and $d_{n}$ be defined in $(3)$. If $\Phi(\zeta) \notin \mathcal{J}_{k}$ for any $k \in\{1,2\}$ and all $\zeta \in \mathcal{K}_{0, R}\left(\zeta_{0}\right)$, then the set $\mathbb{Z}_{k}:=\left\{n \in \mathbb{Z}: d_{n} \notin \mathcal{J}_{k}\right\}$ is nonempty. 
Proof. Since $\Phi$ is a monogenic function in the domain $\mathcal{K}_{0, R}\left(\zeta_{0}\right)$, it can be represented in form (3). Assume, for the sake of contradiction, that $\mathbb{Z}_{k}$ is empty. Then $d_{n}\left(\zeta-\zeta_{0}\right)^{n} \in \mathcal{J}_{k}$ for all $n \in \mathbb{Z}$, which implies that $\Phi(\zeta) \in \mathcal{J}_{k}$ for all $\zeta \in \mathcal{K}_{0, R}\left(\zeta_{0}\right)$. We have a contradiction.

By virtue of Lemma 4.2 , the assumption $\Phi(\zeta) \notin \mathcal{J}_{1} \cup \mathcal{J}_{2}$ for all $\zeta \in \mathcal{K}_{0, R}\left(\zeta_{0}\right)$ implies that both $\mathbb{Z}_{1}$ and $\mathbb{Z}_{2}$ are nonempty. Additionally, we assume that

$$
\mathbb{Z}_{1}, \mathbb{Z}_{2} \text { are bounded from below }
$$

and we set

$$
n_{1}:=\min _{n \in \mathbb{Z}_{1}} n \quad \text { and } \quad n_{2}:=\min _{n \in \mathbb{Z}_{2}} n .
$$

Lemma 4.3. Let $\Phi$ be a monogenic function in $\mathcal{K}_{0, R}\left(\zeta_{0}\right)$. Let assumption (6) hold. Then there exist two monogenic functions $\phi_{1}, \phi_{2}$ in the domain $\mathcal{K}_{R}\left(\zeta_{0}\right)$ and a monogenic function $\psi$ in $\mathcal{K}_{0, R}\left(\zeta_{0}\right)$ such that

$$
\Phi(\zeta)=\left(\zeta-\zeta_{0}\right)^{n_{1}} \phi_{1}(\zeta) I_{1}+\left(\zeta-\zeta_{0}\right)^{n_{2}} \phi_{2}(\zeta) I_{2}+\psi(\zeta) \rho \quad \forall \zeta \in \mathcal{K}_{0, R}\left(\zeta_{0}\right) .
$$

Proof. Using relations (3) and (4), definitions of $\mathbb{Z}_{1}, \mathbb{Z}_{2}$ and assumption (6), we have

$$
\begin{gathered}
\Phi(\zeta)=\sum_{n=-\infty}^{\infty} a_{n}\left(\zeta-\zeta_{0}\right)^{n} I_{1}+\sum_{n=-\infty}^{\infty} b_{n}\left(\zeta-\zeta_{0}\right)^{n} I_{2} \\
\quad+\sum_{n=-\infty}^{\infty}\left((n+1)\left(p_{3} y_{0}+q_{3} z_{0}\right) b_{n+1}+c_{n}\right)\left(\zeta-\zeta_{0}\right)^{n} \rho \\
=\left(\zeta-\zeta_{0}\right)^{n_{1}} \sum_{n \in \mathbb{Z}_{1}} a_{n}\left(\zeta-\zeta_{0}\right)^{n-n_{1}} I_{1}+\left(\zeta-\zeta_{0}\right)^{n_{2}} \sum_{n \in \mathbb{Z}_{2}} b_{n}\left(\zeta-\zeta_{0}\right)^{n-n_{2}} I_{2} \\
\quad+\sum_{n=-\infty}^{\infty}\left((n+1)\left(p_{3} y_{0}+q_{3} z_{0}\right) b_{n+1}+c_{n}\right)\left(\zeta-\zeta_{0}\right)^{n} \rho,
\end{gathered}
$$

where $a_{n}, b_{n}, c_{n}$ are defined in (4). To complete the proof it is natural to set

$$
\begin{gathered}
\phi_{1}(\zeta):=\sum_{n \in \mathbb{Z}_{1}} a_{n}\left(\zeta-\zeta_{0}\right)^{n-n_{1}}, \quad \phi_{2}(\zeta):=\sum_{n \in \mathbb{Z}_{2}} b_{n}\left(\zeta-\zeta_{0}\right)^{n-n_{2}}, \\
\psi(\zeta):=\sum_{n=-\infty}^{\infty}\left((n+1)\left(p_{3} y_{0}+q_{3} z_{0}\right) b_{n+1}+c_{n}\right)\left(\zeta-\zeta_{0}\right)^{n}
\end{gathered}
$$


In the following lemma, we consider some properties of functions $\phi_{1}, \phi_{2}$.

Lemma 4.4. Let assumptions of Lemma 4.3 hold. Moreover, let $\Phi(\zeta) \notin$ $\mathcal{J}_{1} \cup \mathcal{J}_{2}$ for all $\zeta \in \mathcal{K}_{0, R}\left(\zeta_{0}\right)$. Then $\phi_{1}(\zeta), \phi_{2}(\zeta) \notin \mathcal{J}_{1} \cup \mathcal{J}_{2}$ for all $\zeta \in \mathcal{K}_{0, R}\left(\zeta_{0}\right)$.

Proof. We first assume, for the sake of contradiction, that the statement does not hold for $\phi_{1}$. Let there exist $\zeta_{1} \in \mathcal{K}_{0, R}\left(\zeta_{0}\right)$ such that $\phi_{1}\left(\zeta_{1}\right) \in \mathcal{J}_{1}$. Then $\left(\zeta_{1}-\zeta_{0}\right)^{n_{1}} \phi_{1}\left(\zeta_{1}\right) I_{1}=0$, and, by virtue of $(8), \Phi\left(\zeta_{1}\right) \in \mathcal{J}_{1}$. We have a contradiction.

Now, we note that $\phi_{1}$ can be rewritten as

$$
\begin{aligned}
\phi_{1}(\zeta)=\sum_{n \in \mathbb{Z}_{1}} & a_{n}\left(\xi_{1}-\xi_{10}\right)^{n-n_{1}} I_{1}+\sum_{n \in \mathbb{Z}_{1}} a_{n}\left(\xi_{2}-\xi_{20}\right)^{n-n_{1}} I_{2} \\
& +\sum_{n \in \mathbb{Z}_{1}}\left(n-n_{1}\right) a_{n}\left(\xi_{2}-\xi_{20}\right)^{n-n_{1}-1}\left(p_{3}\left(y-y_{0}\right)+q_{3}\left(z-z_{0}\right)\right) \rho .
\end{aligned}
$$

and we assume that there exists $\zeta_{2} \in \mathcal{K}_{0, R}\left(\zeta_{0}\right)$ such that $\phi_{1}\left(\zeta_{2}\right) \in \mathcal{J}_{2}$. This assumption implies that

$$
\sum_{n \in \mathbb{Z}_{1}} a_{n} \delta^{n-n_{1}}=0, \quad \text { where } \quad \delta:=f_{2}\left(\zeta_{2}\right)-\xi_{20} .
$$

Let us consider the set

$$
\mathcal{K}\left(\zeta_{0}, \delta\right):=\left\{\zeta \in \mathcal{K}_{0, R}\left(\zeta_{0}\right):\left|\xi_{1}-\xi_{10}\right|=|\delta|,\left|\xi_{2}-\xi_{20}\right|=|\delta|\right\} .
$$

It is evident that there exists $\tilde{\zeta}_{2} \in \mathcal{K}\left(\zeta_{0}, \delta\right)$ such that $f_{1}\left(\tilde{\zeta}_{2}\right)-\xi_{10}=\delta$. Thus,

$$
\sum_{n \in \mathbb{Z}_{1}} a_{n}\left(f_{1}\left(\tilde{\zeta}_{2}\right)-\xi_{10}\right)^{n-n_{1}}=0
$$

which implies that $\phi_{1}\left(\tilde{\zeta}_{2}\right) \in \mathcal{J}_{1}$, and, by arguing as above, we have a contradiction. Considering $\phi_{2}$ in a similar way as $\phi_{1}$, we deduce the validity of the lemma. $\zeta_{0}$.

In the following lemma we find the logarithmic residue of $\Phi$ at the point

Lemma 4.5. Let $\Phi$ be a monogenic function in $\mathcal{K}_{0, R}\left(\zeta_{0}\right)$ and $\Phi(\zeta) \notin \mathcal{J}_{1} \cup \mathcal{J}_{2}$ for all $\zeta \in \mathcal{K}_{0, R}\left(\zeta_{0}\right)$. Moreover, let $\Phi$ have representation (8), where $\phi_{1}, \phi_{2}$ are monogenic functions in the domain $\mathcal{K}_{R}\left(\zeta_{0}\right)$ and $\psi$ is monogenic in $\mathcal{K}_{0, R}\left(\zeta_{0}\right)$. Then

$$
\frac{1}{2 \pi i} \int_{\Gamma_{r}\left(\zeta_{0}\right)} \Phi^{\prime}(\zeta)(\Phi(\zeta))^{-1} d \zeta=n_{1} I_{1}+n_{2} I_{2}
$$


for an arbitrary $r \in \mathbb{R}$ such that $0<r<R$.

Proof. By Lemma 4.1, the integral on the left hand side of equality (9) exists. Moreover, by Lemma 4.4, $\phi_{1}$ and $\phi_{2}$ do not take values in the ideals of $\mathbb{A}_{2}$ for all $\zeta \in \mathcal{K}_{0, R}\left(\zeta_{0}\right)$, that implies the existence of $\left(\phi_{1}(\zeta)\right)^{-1}$ and $\left(\phi_{2}(\zeta)\right)^{-1}$ for all $\zeta \in \mathcal{K}_{0, R}\left(\zeta_{0}\right)$.

By (8), we immediately have

$$
\begin{gathered}
\Phi^{\prime}(\zeta)=n_{1}\left(\zeta-\zeta_{0}\right)^{n_{1}-1} \phi_{1}(\zeta) I_{1}+\left(\zeta-\zeta_{0}\right)^{n_{1}} \phi_{1}^{\prime}(\zeta) I_{1}+n_{2}\left(\zeta-\zeta_{0}\right)^{n_{2}-1} \phi_{2}(\zeta) I_{2} \\
+\left(\zeta-\zeta_{0}\right)^{n_{2}} \phi_{2}^{\prime}(\zeta) I_{2}+\psi^{\prime}(\zeta) \rho \quad \forall \zeta \in \mathcal{K}_{0, R}\left(\zeta_{0}\right), \quad(10)
\end{gathered}
$$

Taking into account (10) and (11), we obtain

$$
\begin{aligned}
& \frac{1}{2 \pi i} \int_{\Gamma_{r}\left(\zeta_{0}\right)} \Phi^{\prime}(\zeta)(\Phi(\zeta))^{-1} d \zeta=I_{1} \frac{n_{1}}{2 \pi i} \int_{\Gamma_{r}\left(\zeta_{0}\right)}\left(\zeta-\zeta_{0}\right)^{-1} d \zeta \\
& \quad+I_{1} \frac{1}{2 \pi i} \int_{\Gamma_{r}\left(\zeta_{0}\right)} \phi_{1}^{\prime}(\zeta)\left(\phi_{1}(\zeta)\right)^{-1} d \zeta+I_{2} \frac{n_{2}}{2 \pi i} \int_{\Gamma_{r}\left(\zeta_{0}\right)}\left(\zeta-\zeta_{0}\right)^{-1} d \zeta \\
& +I_{2} \frac{1}{2 \pi i} \int_{\Gamma_{r}\left(\zeta_{0}\right)} \phi_{2}^{\prime}(\zeta)\left(\phi_{2}(\zeta)\right)^{-1} d \zeta-\rho \frac{1}{2 \pi i} \int_{\Gamma_{r}\left(\zeta_{0}\right)}\left[\left(\zeta-\zeta_{0}\right)^{-3 n_{2}}\left(\phi_{2}(\zeta)\right)^{-3} \psi(\zeta)\right]^{\prime} d \zeta \\
& =\left(n_{1} I_{1}+n_{2} I_{2}\right) \frac{1}{2 \pi i} \int_{\Gamma_{r}\left(\zeta_{0}\right)}\left(\zeta-\zeta_{0}\right)^{-1} d \zeta+I_{1} \frac{1}{2 \pi i} \int_{\Gamma_{r}\left(\zeta_{0}\right)} \phi_{1}^{\prime}(\zeta)\left(\phi_{1}(\zeta)\right)^{-1} d \zeta \\
& +I_{2} \frac{1}{2 \pi i} \int_{\Gamma_{r}\left(\zeta_{0}\right)} \phi_{2}^{\prime}(\zeta)\left(\phi_{2}(\zeta)\right)^{-1} d \zeta-\rho \frac{1}{2 \pi i} \int_{\Gamma_{r}\left(\zeta_{0}\right)}\left[\left(\zeta-\zeta_{0}\right)^{-3 n_{2}}\left(\phi_{2}(\zeta)\right)^{-3} \psi(\zeta)\right]^{\prime} d \zeta \\
& =:\left(n_{1} I_{1}+n_{2} I_{2}\right) \Lambda_{1}+I_{1} \Lambda_{2}+I_{2} \Lambda_{3}+\rho \Lambda_{4} .
\end{aligned}
$$

By virtue of Theorem A.4 in Appendix A, we have $\Lambda_{1}=1$. Using Theorem A.3 in Appendix A, we obtain the equality $\Lambda_{2}=\Lambda_{3}=0$ because the functions $\phi_{1}^{\prime}(\zeta)\left(\phi_{1}(\zeta)\right)^{-1}$ and $\phi_{2}^{\prime}(\zeta)\left(\phi_{2}(\zeta)\right)^{-1}$ are monogenic in the domain $\mathcal{K}_{R}\left(\zeta_{0}\right)$. Finally, taking into account the continuity of the function $\left(\zeta-\zeta_{0}\right)^{-3 n_{2}}\left(\phi_{2}(\zeta)\right)^{-3} \psi(\zeta)$ on the curve $\Gamma_{r}\left(\zeta_{0}\right)$, we obtain the equality $\Lambda_{4}=$ 0 .

The following result follows from Lemma 4.5. 
Corollary 4.6. Let assumptions of Lemma 4.5 hold. If $n_{1}=n_{2}$ then the logarithmic residue of a monogenic function $\Phi$ at the point $\zeta_{0}$ is an integer.

By virtue of the last paragraph of section 3 and Lemma A.2 in Appendix A, we find it convenient to set

$$
L_{\Phi}\left(\zeta_{0}\right):=\left\{\begin{aligned}
L_{1}\left(\zeta_{0}\right) \cup L_{2}\left(\zeta_{0}\right) \quad & \text { if either } \Phi\left(\zeta_{0}\right) \in \mathcal{R} \text { or } \xi_{10} \text { is } \\
& \text { a non-removable singularity for } F_{1} \text { and } \\
& \xi_{20} \text { is a non-removable singularity for } F_{2}, \\
L_{1}\left(\zeta_{0}\right) \quad & \text { if either } \Phi\left(\zeta_{0}\right) \in \mathcal{J}_{1} \backslash \mathcal{R} \text { or } \xi_{10} \text { is a non-removable } \\
& \text { singularity for } F_{1} \text { and } \xi_{20} \text { is not a non-removable } \\
& \text { singularity for } F_{2}, \\
L_{2}\left(\zeta_{0}\right) & \text { if either } \Phi\left(\zeta_{0}\right) \in \mathcal{J}_{2} \backslash \mathcal{R} \text { or } \xi_{10} \text { is not a non-removable } \\
& \text { singularity for } F_{1} \text { and } \xi_{20} \text { is a non-removable } \\
& \text { singularity for } F_{2} .
\end{aligned}\right.
$$

If $\Phi$ is monogenic in the domain $\mathcal{K}_{0, R}\left(\zeta_{0}\right)$ and, moreover, $\Phi(\zeta) \notin \mathcal{J}_{1} \cup \mathcal{J}_{2}$ for all $\zeta \in \mathcal{K}_{0, R}\left(\zeta_{0}\right)$, and either $\Phi\left(\zeta_{0}\right) \in \mathcal{J}_{1} \cup \mathcal{J}_{2}$ or $\zeta_{0}$ is a non-removable singular point of $\Phi$, we call $\zeta_{0}$ by a singular point of the logarithmic derivative of function $\Phi$. Obviously, if $\zeta_{0}$ is a such point, every point of $\mathcal{K}_{R}\left(\zeta_{0}\right) \cap L_{\Phi}\left(\zeta_{0}\right)$ is a singular point of the logarithmic derivative of $\Phi$. In addition, if assumption (6) holds, we call a hypercomplex number $n_{1} I_{1}+n_{2} I_{2}$ by the singularity index of the logarithmic derivative of function $\Phi$ at the point $\zeta_{0}$.

For an arbitrary set $\Omega \subset E_{3}$, we find it reasonable to set

$$
\begin{aligned}
& \mathcal{S}_{\Phi}(\Omega):=\{\zeta \in \Omega: \zeta \text { is a non-removable singularity of } \Phi\}, \\
& \mathcal{J}_{\Phi}(\Omega):=\left\{\zeta \in \Omega: \Phi(\zeta) \in \mathcal{J}_{1} \cup \mathcal{J}_{2}\right\} .
\end{aligned}
$$

Let $G \subset \Pi(\zeta)$ be a domain in $\Pi(\zeta)$. Then $\operatorname{cl} G$ and $\partial G$ denote the closure and the boundary of $G$ in the induced topology of $\Pi(\zeta)$, respectively.

Now, we can formulate the following theorem on the sum of logarithmic residues for monogenic functions taking values in the algebra $\mathbb{A}_{2}$.

Theorem 4.7. Let $\Omega$ be a domain in $E_{3}$ and $\Phi$ be a monogenic function in $\Omega \backslash \mathcal{S}_{\Phi}(\Omega)$. Let $\zeta_{0}$ be an arbitrary point in $\Omega$. Let $G \subset \Pi\left(\zeta_{0}\right)$ be a domain in $\Pi\left(\zeta_{0}\right)$ such that $\mathrm{cl} G \subset \Omega$ and $\partial G$ be a closed Jordan rectifiable curve. Let $\partial G$ do not contain singular points of the logarithmic derivative of function $\Phi, \mathcal{S}_{\Phi}(G) \cup \mathcal{J}_{\Phi}(G)=\left\{\zeta_{k}\right\}_{k=1}^{m}$, where $m$ is finite, and there exist $R>0$ such that assumption (6) holds in $\mathcal{K}_{0, R}\left(\zeta_{k}\right)$ for all $k=1,2, \ldots, m$. Moreover, let $n_{1 k} I_{1}+n_{2 k} I_{2}$ denoting the singularity index of the logarithmic derivative of function $\Phi$ at the point $\zeta_{k}$ be finite for all $k=1,2, \ldots, m$. Then

$$
\frac{1}{2 \pi i} \int_{\Gamma} \Phi^{\prime}(\zeta)(\Phi(\zeta))^{-1} d \zeta=\sum_{k=1}^{m}\left(n_{1 k} I_{1}+n_{2 k} I_{2}\right)
$$


where $\Gamma$ is an arbitrary closed Jordan rectifiable curve in the domain $\Omega \backslash\left(\mathcal{S}_{\Phi}(\Omega) \cup \mathcal{J}_{\Phi}(\Omega)\right)$ which is homotopic to $\partial G$ in this domain.

Proof. Let positive $R$ be such that the sets $\mathcal{K}_{0, R}\left(\zeta_{k}\right) \subset \Omega$ are pairwise disjoint for all $k=1,2, \ldots, m$. Since $\Gamma$ is homotopic to $\partial G$ in $\Omega \backslash\left(\mathcal{S}_{\Phi}(\Omega) \cup \mathcal{J}_{\Phi}(\Omega)\right)$, by Theorem A.3 in Appendix A, we have

$$
\begin{gathered}
\frac{1}{2 \pi i} \int_{\Gamma} \Phi^{\prime}(\zeta)(\Phi(\zeta))^{-1} d \zeta=\frac{1}{2 \pi i} \int_{\partial G} \Phi^{\prime}(\zeta)(\Phi(\zeta))^{-1} d \zeta \\
=\frac{1}{2 \pi i} \sum_{k=1}^{m} \int_{\Gamma_{r}\left(\zeta_{k}\right)} \Phi^{\prime}(\zeta)(\Phi(\zeta))^{-1} d \zeta
\end{gathered}
$$

for any $r, 0<r<R$.

Now, to complete the proof one can apply Lemma 4.5.

The following theorem is an analogue of the known result in complex analysis.

Theorem 4.8. Let assumptions of Theorem 4.7 hold. Then

$$
\frac{1}{2 \pi i} \int_{\Gamma} \Phi^{\prime}(\zeta)(\Phi(\zeta))^{-1} d \zeta=\left(N_{F_{1}}-P_{F_{1}}\right) I_{1}+\left(N_{F_{2}}-P_{F_{2}}\right) I_{2},
$$

where $N_{F_{k}}, P_{F_{k}}$ are the numbers of zeros and poles, respectively, of the function $F_{k}$ in the domain $D_{k}(G)$ for $k=1,2$.

Proof. Since $\Gamma$ is homotopic to $\partial G$ in $\Omega \backslash\left(\mathcal{S}_{\Phi}(\Omega) \cup \mathcal{J}_{\Phi}(\Omega)\right)$, we conclude that $\Gamma$ does not contain singularities of the logarithmic derivative of function $\Phi$. Then the following equality is true:

$$
\frac{1}{2 \pi i} \int_{\Gamma} \Phi^{\prime}(\zeta)(\Phi(\zeta))^{-1} d \zeta=\frac{1}{2 \pi i} \int_{\partial G} \Phi^{\prime}(\zeta)(\Phi(\zeta))^{-1} d \zeta=\frac{1}{2 \pi i} \triangle_{\partial G} \ln \Phi(\zeta),
$$

where $\triangle_{\partial G} \ln \Phi(\zeta)$ denotes the increment of function $\ln \Phi(\zeta)$ as $\zeta$ passes the curve $\partial G$. Equalities (1) and (2) yield the equality

$$
\ln \Phi(\zeta)=\ln F_{1}\left(\xi_{1}\right) I_{1}+\ln F_{2}\left(\xi_{2}\right) I_{2}+\frac{\left(p_{3} y+q_{3} z\right) F_{2}^{\prime}\left(\xi_{2}\right)+F_{0}\left(\xi_{2}\right)}{F_{2}\left(\xi_{2}\right)} \rho
$$

for all $\zeta \in \Gamma$, where $\xi_{1} \in D_{1}(\partial G), \xi_{2} \in D_{2}(\partial G)$.

Since $\Phi$ does not take values in the ideals on the curve $\partial G$, by virtue of (2), we conclude that the function $F_{2}$ is not equal to zero on the curve $D_{2}(\partial G)$ in 
the complex plane. Therefore, the function $\left(\left(p_{3} y+q_{3} z\right) F_{2}^{\prime}\left(\xi_{2}\right)+F_{0}\left(\xi_{2}\right)\right) / F_{2}\left(\xi_{2}\right)$ is continuous on the curve $D_{2}(\partial G)$ and, hence, its increment in passing this curve is equal to zero.

Thus, $\triangle_{\partial G} \ln \Phi(\zeta)=\triangle_{D_{1}(\partial G)} F_{1}\left(\xi_{1}\right) I_{1}+\triangle_{D_{2}(\partial G)} F_{2}\left(\xi_{2}\right) I_{2}$ and, in view of the principle of argument for analytic functions of a complex variable (see, e.g., [18, sec. 10]), equality (14) is transformed into (13).

Finally, we formulate the result which follows from Theorem 4.8.

Corollary 4.9. Let assumptions of Lemma 4.5 hold. Let functions $F_{1}$, $F_{2}$ be as in (2). Then the logarithmic residue of a monogenic function $\Phi$ at the point $\zeta_{0}$ is an integer if and only if the logarithmic residue of $F_{1}$ at the point $\xi_{10}$ and the logarithmic residue of $F_{2}$ at the point $\xi_{20}$ coincide. If so, the logarithmic residues of all these functions coincide.

\section{A Appendix}

In the Appendix, we collect some results exploited in the article.

Lemma A.1. Let $\zeta_{0} \in E_{3}$ and $0 \leq r<R$. Then the domain $\mathcal{K}_{r, R}\left(\zeta_{0}\right)$ can be represented as a union of domains $\widetilde{\mathcal{K}}_{r, R}\left(\zeta_{0}\right)$ and $\widehat{\mathcal{K}}_{r, R}\left(\zeta_{0}\right)$, each of them is convex in both directions $L_{1}$ and $L_{2}$, and $\widetilde{\mathcal{K}}_{r, R}\left(\zeta_{0}\right) \cap \widehat{\mathcal{K}}_{r, R}\left(\zeta_{0}\right)$ is an open set.

Proof. The proof is constructive. We first note that the case $L_{1}=L_{2}$ is trivial. It is enough to set $\widetilde{\mathcal{K}}_{r, R}\left(\zeta_{0}\right)=\widehat{\mathcal{K}}_{r, R}\left(\zeta_{0}\right)=\mathcal{K}_{r, R}\left(\zeta_{0}\right)$.

Next, we suppose that $L_{1}$ and $L_{2}$ do not coincide. Let $\Pi^{*}\left(\zeta_{0}\right):=\left\{\zeta_{0}+t_{1} e_{1}^{*}+t_{2} e_{2}^{*}: t_{1}, t_{2} \in \mathbb{R}\right\}$ be a plane in $E_{3}$. If $r \neq 0$, we take two planes $\Pi^{*}\left(\zeta_{0}+r \hat{e}_{1}^{*}\right)$ and $\Pi^{*}\left(\zeta_{0}-r \hat{e}_{1}^{*}\right)$. These planes split $\mathcal{K}_{r, R}\left(\zeta_{0}\right)$ into six parts, four of them are located between the planes. One can uniquely indicate two pairs of sets formed by the sets from the mentioned four parts such that the union of two sets of a pair is convex in both directions $L_{1}$ and $L_{2}$. We denote these pair by $\Omega_{1}$ and $\Omega_{2}$. Then, we set

$$
\begin{aligned}
& \widetilde{\mathcal{K}}_{r, R}\left(\zeta_{0}\right):=\mathcal{K}_{r, R}\left(\zeta_{0}\right) \backslash \bar{\Omega}_{1}, \\
& \widehat{\mathcal{K}}_{r, R}\left(\zeta_{0}\right):=\mathcal{K}_{r, R}\left(\zeta_{0}\right) \backslash \bar{\Omega}_{2},
\end{aligned}
$$

where $\bar{\Omega}_{k}$ denotes the closure of $\Omega_{k}$ in the induced topology of $E_{3}, k \in\{1,2\}$. By the construction, it easy to see that both $\widetilde{\mathcal{K}}_{r, R}\left(\zeta_{0}\right)$ and $\widehat{\mathcal{K}}_{r, R}\left(\zeta_{0}\right)$ are open

and convex in directions $L_{1}, L_{2}$ sets. As a conclusion, $\widetilde{\mathcal{K}}_{r, R}\left(\zeta_{0}\right) \cap \widehat{\mathcal{K}}_{r, R}\left(\zeta_{0}\right)$ is an open set.

If $r=0$, we take one plane $\Pi\left(\zeta_{0}\right)$ and split it into four parts by the straight lines $L_{1}\left(\zeta_{0}\right)$ and $L_{2}\left(\zeta_{0}\right)$. By the same arguing as above, we deduce the validity of the lemma. 
The following lemma is the immediate consequence of Lemma 1 in [14].

Lemma A.2. Let $\Omega$ be a convex in directions $L_{1}, L_{2}$ domain in $E_{3}$. Let $\Phi$ be a monogenic function in $\Omega$. Let $k \in\{1,2\}$. Let $\zeta_{0}$ be an arbitrary point of $\Omega$. Then $\Phi\left(\zeta_{0}\right) \in \mathcal{J}_{k}$ implies $\Phi(\zeta) \in \mathcal{J}_{k}$ for all $\zeta \in \Omega \cap L_{k}\left(\zeta_{0}\right)$.

The following result is an analogue of the Cauchy integral theorem for monogenic functions in $\mathbb{A}_{2}$. We note that a more general result can be found in $[19$, Thm. 4.2].

Theorem A.3. Let $\Omega$ be a convex in directions $L_{1}, L_{2}$ domain in $E_{3}$ and $\Phi: \Omega \rightarrow \mathbb{A}_{2}$ be a monogenic function in $\Omega$. Let $\gamma \subset \Omega$ be a closed Jordan rectifiable curve which is homotopic to a point of $\Omega$. Then $\int_{\gamma} \Phi(\zeta) d \zeta=0$.

The following statement is a particular case of Theorem 6.1 in [19].

Theorem A.4. Let $\Omega$ be a convex in directions $L_{1}, L_{2}$ domain in $E_{3}$. Let $\Phi: \Omega \rightarrow \mathbb{A}_{2}$ be a monogenic function in $\Omega$. Then for every point $\zeta_{0} \in \Omega$ the following equality is true:

$$
\Phi\left(\zeta_{0}\right)=\frac{1}{2 \pi i} \int_{\gamma} \Phi(\zeta)\left(\zeta-\zeta_{0}\right)^{-1} d \zeta,
$$

where $\gamma$ is a closed Jordan rectifiable curve in $\Omega$, that surrounds once the set $L_{1}\left(\zeta_{0}\right) \cup L_{2}\left(\zeta_{0}\right)$.

\section{Acknowledgements}

The author R. Pukhtaievych was supported by Cariparo Foundation. This research was also partially supported by Ministry of Education and Science of Ukraine (ProjectNo. 0116U001528).

\section{References}

[1] Mittenthal L. Operator valued analytic functions and generalizations of spectral theory, Pacific J. Math., 24, 1968, pp. 119-132.

[2] Bart H., Ehrhardt T. And Silbermann B. Logarithmic residues in Banach algebras, Integr. Equ. Oper. Theory, 19(2), 1994, pp. 135-152.

[3] Bart H., Ehrhardt T. and Silbermann B. Logarithmic residues in the Banach algebra generated by the compact operators and the identity, Math. Nachr., 268(1), 2004, pp. 3-30.

[4] Bart H., Ehrhardt T. and Silbermann B. Sums of idempotents and logarithmic residues in zero pattern matrix algebras, Linear Algebra Appl., 498, 2016, pp. 262-316. 
[5] Grishchuk S.V. And Plaksa S.A. On the logarithmic residues of monogenic functions of a biharmonic variable, Zb. Pr. Inst. Mat. NAN Ukr., 7(2), 2010, pp. 227-234. (in Russian)

[6] Plaksa S.A. And ShPakivskyi V.S. On the logarithmic residues of monogenic functions in three-dimensional harmonic algebra with twodimensional radical, Ukr. Math. J., 65(7), 2013, pp. 1079-1086.

[7] BART H. Spectral properties of locally holomorphic vector-valued functions, Pacific J. Math., 52(2), 1974, pp. 321-329.

[8] Bart H., Ehrhardt T. And Silbermann B. Logarithmic residues, generalized idempotents and sums of idempotents in Banach algebras, Integral Equations Operator Theory, 29(2), 1997, pp. 155-186.

[9] Taylor A.E. and Lay D.C. Introduction to Functional Analysis, Second Edition, John Wiley and Sons, New York, 1980.

[10] Aizenberg L.A. And Yuzhakov A.P. Integral representations and residues in multidimensional complex analysis, American Mathematical Society, Providence, RI, 1983.

[11] Aizenberg L.A., Tsikh A.K. And Yuzhakov A.P. Multidimensional Residues and Applications, in Several Complex Variables II, (Khenkin G.M. and Vitushkin A.G., eds.), Encyclopaedia of Math. Sc. 8, Springer-Verlag, Berlin, Heidelberg, New-York, 1990, pp. 1-58.

[12] Tsikn A.K. Multidimensional Residues and Their Applications, Nauka, Novosibirsk, 1988; English transl., Translations of Mathematical Monographs 103, Amer. Math. Soc., Providence, 1992.

[13] LoRCH E. R. The theory of analytic function in normed abelian vector rings, Trans. Amer. Math. Soc., 54, 1943, pp. 414-425.

[14] Plaksa S.A. and Pukhtaievych R.P. Constructive description of monogenic functions in a three-dimensional harmonic algebra with onedimensional radical, Ukr. Math. J., 65(5), 2013, pp. 740-751.

[15] Shpakivsky V.S. Constructive description of monogenic functions in a finite-dimensional commutative associative algebra, Adv. Pure Appl. Math., 7(1), 2016, pp. 63-75.

[16] Pukhtaievych R.P. Power series and Laurent series in threedimensional harmonic algebra with a one-dimensional radical, $\mathrm{Zb}$. Pr. Inst. Mat. NAN Ukr., 9(2), 2012, pp. 311-326. (in Russian) 
[17] ShPakivskyi V.S. Power series and Laurent series in three-dimensional harmonic algebra with a two-dimensional radical, Zb. Pr. Inst. Mat. NAN Ukr., 7(2), 2010, pp. 314-321. (in Russian)

[18] Rudin W. Real and Complex Analysis, Mc Graw Hill, New York, third ed., 1987.

[19] ShPAKIVSKYI V.S. Curvilinear integral theorems for monogenic functions in commutative associative algebras, Adv. Appl. Clifford Algebras, 26, 2016, pp. 417-434.

Roman Pukhtaievych,

Department of Mathematics "Tullio Levi-Civita",

University of Padova,

63, Via Trieste, Padova, Italy

Email: roman@math.unipd.it

rpukhtaievych@gmail.com

Sergiy Plaksa,

Department of complex analysis and potential theory,

Institute of Mathematics of the National Academy of Sciences of Ukraine,

3, Tereshchenkivska st., Kiev, Ukraine

Email: plaksa@imath.kiev.ua

plaksa62@gmail.com 